\title{
Continuity in Mechanism Design without Transfers ${ }^{1}$
}

\author{
David Martimort ${ }^{2}$ and Aggey Semenov ${ }^{3}$
}

This Version: March 16, 2006

\begin{abstract}
We adopt a mechanism design approach to model communication between a principal and a privately informed agent in the context where monetary incentives are not available. We provide a simple condition on the distribution of the agent's type which ensures that the optimal mechanism is continuous. With strict log-concavity of the distribution, there exists a unique optimal mechanism that is characterized.
\end{abstract}

Keywords: Communication, Mechanism Design without Transfers.

JEL Classification: D72; D82.

\section{Introduction}

Mechanism design problems between a principal and an agent whose preferences over a state-contingent policy conflict in contexts where monetary transfers are not available have recently received a lot of attention. Such models have been developed in view of applications to delegation within the firm (Holmström (1984)), political science (Baron (2000) and Martimort and Semenov (2006)), or regulation (Armstrong (1994)) among others. Those models have provided insights on the pattern of communication which takes place within these organizations. With a mechanism design perspective, the principal

\footnotetext{
${ }^{1}$ We thank Vianney Dequiedt for comments.

${ }^{2}$ University of Toulouse, (IDEI, GREMAQ) and Institut Universitaire de France.Corresponding author: martimor@cict.fr. Phone:(33) 5611286 14. Fax: (33) 5611286 37. Address: IDEI, Manufacture des Tabacs, Bt F., 21, Allée de Brienne, 31000 Toulouse, France.

${ }^{3}$ University of Toulouse (GREMAQ) and CORE.
} 
commits to a policy rule before the agent reports his private information on the state of the world. ${ }^{1}$ As such, this approach can be viewed as complementary of the huge literature which, following Crawford and Sobel (1982), deals instead with the reverse signaling timing.

The motivation for the commitment assumption is twofold. From a practical viewpoint first, this assumption might seem sometimes at least as reasonable as the assumption made in the signaling literature that the informed agent moves first. In the context of the organization of legislative committees, for instance, the legislature (principal) can structure the work of committees by moving first. The timing of the mechanism design approach seems also a relatively good short-cut for modeling the repeated relationship between this legislature and a particular committee. More importantly maybe, the commitment assumption is also attractive because it solves the equilibrium indeterminacy that arises in the signaling environment where the agent moves first. The mechanism design approach allows also a full characterization of communication patterns achievable at any equilibrium of a communication game among the agents and allows a meaningful optimization. This is important from a normative viewpoint.

It turns out that in the simple environments with quadratic single-peaked preferences inherited from Crawford and Sobel (1982), the structure of deterministic incentive mechanisms is relatively straightforward as demonstrated by Melumad and Shibano (1991). Those mechanisms may nevertheless exhibit discontinuities much in lines with the kind of discontinuities which arise with the partition equilibria of signaling models à la Crawford and Sobel (1982). Those discontinuities stand however in sharp contrast with the findings of Moulin (1980). This author indeed characterized dominant strategy incentive mechanisms when no parametric restriction on the domain of single-peaked preferences is imposed and found that they are continuous. Without restriction on preferences, the differentiable approach used below ${ }^{2}$ is no longer available and Moulin's characterization in fact eliminates discontinuous mechanisms of interest.

In practice however, much attention has been given to continuous mechanisms. ${ }^{3}$ For instance, Baron (2000, p. 501) directly postulated the continuity of the optimal communication mechanism in comparing the performances of a legislature using transfers and those of a legislature constrained in this respect. Martimort and Semenov (2006) used continuous communication schemes to compare the performance of different organizations of the legislature with several committees. The benefit of focusing on continuous mechanisms is of course a gain in tractability; an important step to proceed to a normative

\footnotetext{
${ }^{1}$ In some of the above applications, the direct mechanism can equivalently be implemented through delegation of the decision to the agent under some constraints.

${ }^{2}$ As well as in Melumad and Shibano (1991).

${ }^{3}$ Or to their interpretation as connected delegation sets.
} 
analysis comparing communication patterns and payoffs across organizational modes. A key issue is thus to determine how much is lost, if anything, by making such restriction. In case continuity of the mechanism is not a significant restriction, the signaling model with its partition equilibria and the screening model significantly differ, leaving as a puzzle which approach is actually the best.

This note provides a sufficient condition that can be directly checked on the distribution of the agent's type which ensures that the optimal mechanisms is indeed continuous. ${ }^{4}$ Adding then strict log-concavity of the distribution, we prove that there exists a unique optimal mechanism which corresponds to a partial delegation of the right to make a decision to the agent.

\section{The Model}

We consider a mechanism design problem without transfers between an agent and a principal. The agent and the principal have single-peaked quadratic preferences with respectively ideal points $\theta$ and $\theta+\delta$ defined as follows:

$$
U(q, \theta)=-\frac{1}{2}(q-\theta)^{2}, \text { and } V(q, \theta)=-\frac{1}{2}(q-\theta-\delta)^{2}
$$

where the policy $q$ is one-dimensional and $\delta>0$ represents the bias of the principal.

The agent has private information on his ideal point $\theta$. This parameter is drawn on a set $\Theta=[\underline{\theta}, \bar{\theta}] \subset \mathbb{R}$ according to the cumulative distribution $F(\cdot)$ which has an atomless and everywhere positive density $f=F^{\prime}$. We assume that $f(\cdot)$ is continuous and almost everywhere itself differentiable.

To model communication between the principal and his agent, we follow a mechanism design approach. The principal can precommit himself to a policy rule stipulating which decision should be made as a function of the agent's report on the state of nature. From the Revelation Principle, there is indeed no loss of generality in restricting the principal to offer a direct revelation mechanism $\{q(\hat{\theta})\}_{\hat{\theta} \in \Theta}$ which is truthful.

The timing of the contractual game is as follows. First, the agent observes his ideal point. Second, the principal offers a mechanism $\{q(\hat{\theta})\}_{\hat{\theta} \in \Theta}$. Third, the agent reports his preferences to the principal. Fourth, the corresponding policy is implemented according to the rule to which the principal has precommitted.

\footnotetext{
${ }^{4}$ Our result on continuity appeared in an earlier working paper "Communication by Multiple Interest Groups" (2005). Independently, Alonso and Matouschek (2005) have generalized those conditions taking into account that the principal's utility function may not be quadratic. Our proof is more elementary and direct than theirs.
} 


\section{Incentive Compatibility}

Incentive compatibility constraints for the agent are written as:

$$
-\frac{1}{2}(q(\theta)-\theta)^{2} \geq-\frac{1}{2}(q(\hat{\theta})-\theta)^{2} \quad \forall(\theta, \hat{\theta}) \in \Theta^{2} .
$$

With our assumption of quadratic preferences, the general structure of the set of incentive compatible mechanisms can be easily derived following the work of Melumad and Shibano (1991).

Proposition 1 : (Melumad and Shibano $\left.(1991)^{5}\right)$ An incentive compatible scheme $q(\cdot)$ must satisfy the following conditions:

- $q(\theta)$ is weakly increasing and thus almost everywhere differentiable;

- if $q(\theta)$ is strictly increasing, $q(\theta)=\theta$;

- if $q(\theta)$ is discontinuous at a point $\theta_{1}$ then:

$$
\begin{aligned}
& \text { - } \quad q\left(\theta_{1}^{+}\right)+q\left(\theta_{1}^{-}\right)=2 \theta_{1}, \\
& \text { - } \quad q(\theta) \text { is flat on the right and the left of } \theta_{1}, \\
& \text { - } \quad q\left(\theta_{1}\right) \text { belongs to the pair }\left\{q\left(\theta_{1}^{-}\right), q\left(\theta_{1}^{+}\right)\right\} .
\end{aligned}
$$

The proof is instructive because it yields some insights on the nature of incentive compatible schemes. At any differentiability point of $q(\cdot)$, we must indeed have:

$$
(q(\theta)-\theta) \dot{q}(\theta)=0
$$

This incentive constraint is satisfied by two interesting classes of schemes: the pooling ones where $q(\theta)=q$ on all $\Theta$, and the fully separating one corresponding to the agent's ideal point, $q(\theta)=\theta$ on all $\Theta$. The optimal mechanism will in fact be a compromise between such schemes.

From Proposition 1, continuous mechanisms have a simple form in this environment. It can be easily seen that they have at most one strictly increasing part. Typically, let us denote $\hat{\theta}_{1}$ and $\hat{\theta}_{2}$ the boundary of the segment where $q(\theta)=\theta$, a continuous scheme is of the following kind:

$$
q(\theta)=\min \left\{\hat{\theta}_{2}, \max \left\{\theta, \hat{\theta}_{1}\right\}\right\} .
$$

This is nothing else than the minmax rule due to Moulin (1980).

\footnotetext{
${ }^{5}$ Melumad and Shibano (1991) have proved this result for general single-peaked utility functions satisfying the single-crossing property. We get in fact a shorter proof by specializing to the quadratic case.
} 


\section{Continuity of the Optimal Mechanism}

Next Proposition provides a sufficient condition on the distribution of types to ensure that the optimal mechanism is actually continuous with quadratic preferences.

Proposition 2 : The optimal mechanism is continuous if ${ }^{6}$

$$
f(\theta)-\delta f^{\prime}(\theta) \geq 0 \text { for almost all } \theta .
$$

Proposition 2 shows that, when (5) holds, any scheme with a discontinuity at a point $\theta$ is dominated by a continuous scheme which follows the most preferred policy of the agent on an interval around $\theta$.

Proposition 2 is useful to directly check on distributions the continuity of the optimal mechanism. Condition (5) is weaker than requiring the concavity of the cumulative distribution $F(\cdot){ }^{7}$ It is harder to satisfy when distributions may have a sharp increase at some point.

We are now ready to give a simple characterization of the optimal mechanism.

Proposition 3 : Assume that condition (5) holds, and that the distribution $F(\cdot)$ is strictly log-concave. When the conflict between the principal and the agent is not too severe, i.e., $\delta<\int_{\underline{\theta}}^{\bar{\theta}} F(y) d y=\bar{\theta}-E_{f}(\theta)$, there exists a unique optimal mechanism which is of the form

$$
q(\theta)=\max \{\theta, \hat{\theta}\},
$$

where the cut-off $\hat{\theta}$ is uniquely defined by the condition

$$
\delta=\frac{1}{F(\hat{\theta})} \int_{\underline{\theta}}^{\hat{\theta}} F(\theta) d \theta=\hat{\theta}-E_{f}(\theta \mid \theta \leq \hat{\theta}) .
$$

When $\delta \geq \int_{\underline{\theta}}^{\bar{\theta}} F(\theta) d \theta$, there is no communication at the optimal mechanism.

Proposition 3 shows that the principal always benefits from communication on the upper tail of the distribution, whereas pooling is instead preferred on the lower tail. The intuition is the following. First, we know that a continuous mechanism has at most one interval where effective communication takes place between the principal and the agent (see (4)). Clearly, having a pooling mechanism on an upper tail $\theta \geq \hat{\theta}_{2}$ cannot be optimal.

\footnotetext{
${ }^{6}$ For $\theta=\underline{\theta}$ (resp. $(\theta=\bar{\theta})$, we take the right (resp. left)-derivative.

${ }^{7} \mathrm{~A}$ special case being given by the uniform distribution.
} 
Indeed, the principal can offer an incentive scheme with effective communication inducing a policy $q(\theta)=\theta$ for $\theta \geq \hat{\theta}_{2}$ which is closer to his own ideal point $q^{*}(\theta)=\theta+\delta$ than any pooling scheme $q(\theta)=\hat{\theta}_{2}$ when $\theta$ is large enough. Hence, communication is valuable on the upper tail. Second, consider now the lower tail of the type distribution. The principal prefers to offer a pooling policy on such an interval. The cost of doing so is of course that the policy does not depend on $\theta$. The benefit is that, for the highest values of $\theta$ on that interval, this pooling policy is closer on average to the principal's ideal point. This benefit may exceed the cost borne on the very lowest values with a pooling scheme. This is so when relatively little weight is left to that lower tail. Log-concavity ensures this last property.

Proposition 3 highlights a fundamental trade-off between rigidity on the lower tail and flexibility on the upper tail of the types distribution. The cut-off value $\hat{\theta}$ summarizes how those two forces compensate each other. The alternative expression in (6) reinforces our understanding of this trade-off. On the lower tail, the principal chooses a policy $\delta+E_{f}(\theta \mid \theta \leq \hat{\theta})$, whereas on the upper tail, the agent has all discretion in choosing the policy. The cut-off is determined by making the principal just indifferent between these two options.

Example: For a uniform distribution on $[\underline{\theta}, \bar{\theta}]$, we have

$$
\delta=\frac{1}{\hat{\theta}-\underline{\theta}} \int_{\underline{\theta}}^{\hat{\theta}}(\theta-\underline{\theta}) d \theta=\frac{\hat{\theta}-\underline{\theta}}{2},
$$

so that communication never takes place when $\delta \geq \frac{\Delta \theta}{2}$.

\section{References}

Alonso, R. and N. Matouschek, 2005, "Optimal Delegation", Mimeo Norwestern.

Armstrong, M., 1994, "Delegation and Discretion", Discussion Papers in Economics and Econometrics, $n^{0} 9421$, University of Southampton.

Baron, D., 2000, "Legislative Organization with Informational Committees", American Journal of Political Sciences, 44: 485-505.

Crawford, V. and J. Sobel, 1982, "Strategic Information Transmission", Econometrica, 50: 1431-1451.

Holmström, B. 1984, "On the Theory of Delegation", in M. Boyer and R. Kihlstrom; (eds.), Bayesian Models in Economic Theory, Elsevier Science Publishers B.V. 
Martimort, D. and A. Semenov, 2006, "Competition and Collusion between Interest Groups in Legislative Politics", Mimeo IDEI.

Melumad, N. and T. Shibano, 1991, "Communication in Settings with no Transfers", Rand Journal of Economics, 22: 173-198.

Moulin, H., 1980, "On Strategy-Proofness and Single-Peakedness", Public Choice, 35: 437-455.

\section{Appendix}

- Proof of Proposition 1: From standard revealed preferences arguments, we have indeed for any $(\theta, \hat{\theta}) \in \Theta^{2}$ such that $\theta>\hat{\theta}$ :

$$
-\frac{1}{2}(q(\theta)-\theta)^{2} \geq-\frac{1}{2}(q(\hat{\theta})-\theta)^{2}, \text { and }-\frac{1}{2}(q(\hat{\theta})-\hat{\theta})^{2} \geq-\frac{1}{2}(q(\theta)-\hat{\theta})^{2} .
$$

Summing those two constraints yields

$$
(\theta-\hat{\theta})(q(\theta)-q(\hat{\theta})) \geq 0 .
$$

Therefore, $q(\cdot)$ is weakly increasing and thus almost everywhere differentiable.

At any point of differentiability, we must have (3) and thus $q(\theta)$ is either flat or $q(\theta)=\theta$, i.e., corresponds to the most-preferred choice of the lobbying group.

If $q(\cdot)$ is discontinuous at $\theta_{1}, q\left(\theta_{1}^{+}\right) \neq q\left(\theta_{1}^{-}\right)$, but type $\theta_{1}$ must be indifferent between choosing the policies which are respectively on the left and on the right of $\theta_{1}$

$$
-\frac{1}{2}\left(q\left(\theta_{1}^{-}\right)-\theta_{1}\right)^{2}=-\frac{1}{2}\left(q\left(\theta_{1}^{+}\right)-\theta_{1}\right)^{2}
$$

Using the right- and left-hand sides, we then get (2).

Because $q\left(\theta_{1}^{+}\right) \neq q\left(\theta_{1}^{-}\right)$at a discontinuity and $q(\cdot)$ is differentiable on both sides, it cannot be that $q\left(\theta_{1}\right)$ is not flat on those sides.

Finally, (A1) shows that either $q\left(\theta_{1}\right)=q\left(\theta_{1}^{+}\right)$or $q\left(\theta_{1}\right)=q\left(\theta_{1}^{-}\right)$.

- Proof of Proposition 2: Suppose, to the contrary, that the optimal mechanism $q(\theta)$ is discontinuous at $x \in[\underline{\theta}, \bar{\theta}]$. Then using the structure of the incentive compatible mechanisms given in Proposition 1 , there exists $\Delta>0$ such that on the interval $[x-$ $\Delta, x+\Delta]$ the contract $q(\theta)$ has the form: ${ }^{8}$

$$
q(\theta)= \begin{cases}x+\Delta & \text { if } \theta \in(x, x+\Delta] \\ x-\Delta & \text { if } \theta \in[x-\Delta, x)\end{cases}
$$

\footnotetext{
${ }^{8}$ We take the interval $[x-\Delta, x+\Delta] \subset[\underline{\theta}, \bar{\theta}]$. The cases when $x-\Delta<\underline{\theta}$ or $x+\Delta>\bar{\theta}$ are treated similarly.
} 
and $q(x) \in\{x-\Delta, x+\Delta\}$.

We can consider the new mechanism $q(\theta, \varepsilon)$ which is parameterized by $\varepsilon \in[0, \Delta]$, and which differs from $q(\theta)$ only in $[x-\Delta, x+\Delta]$ where it is defined by: ${ }^{9}$

$$
q(\theta, \varepsilon)= \begin{cases}\theta & \text { if } \theta \in[x+\Delta-\varepsilon, x+\Delta) \\ x+\Delta-\varepsilon & \text { if } \theta \in(x, x+\Delta-\varepsilon] \\ x-\Delta+\varepsilon & \text { if } \theta \in[x-\Delta+\varepsilon, x) \\ \theta & \text { if } \theta \in[x-\Delta, x-\Delta+\varepsilon] .\end{cases}
$$

This mechanism preserves incentive compatibility. The derivative of the principal's expected payoff with respect to $\varepsilon$ evaluated at $\varepsilon=0$ is positive when: ${ }^{10}$

$$
\delta(2 F(x)-F(x-\Delta)-F(x+\Delta))-\int_{x-\Delta}^{x} F(y) d y+\int_{x}^{x+\Delta} F(y) d y \geq 0 .
$$

When condition (A2) holds at any $x$ and for any $\Delta$, a discontinuous mechanism can be improved upon. A contradiction.

Suppose now that $F(\cdot)$ is twice differentiable. Using Taylor expansions for $\Delta$ small enough, (A2) yields:

$$
f(x)-\delta f^{\prime}(x) \geq 0 .
$$

Reciprocally, fix any $x$ and $\Delta>0$ and for $t \in[0, \Delta]$ define

$$
\phi(t)=\delta(2 F(x)-F(x-t)-F(x+t))-\int_{x-t}^{x} F(y) d y+\int_{x}^{x+t} F(y) d y .
$$

Notice then that

$$
\phi^{\prime}(t)=\delta(f(x-t)-f(x+t))-F(x-t)+F(x+t)
$$

with, $\phi^{\prime}(\cdot)$ continuous, $\phi^{\prime}(0)=0$ and

$$
\phi^{\prime \prime}(t)=-\delta f^{\prime}(x-t)+f(x-t)-\delta f^{\prime}(x+t)+f(x+t) \geq 0
$$

when (A3) holds. Hence, $\phi^{\prime}(t) \geq 0$ and $\phi(t) \geq 0$ for all $t \in[0, \Delta]$.

- Proof of Proposition 3: Note that the principal's expected payoff with a continuous scheme characterized by the cut-offs $\hat{\theta}_{1}$ and $\hat{\theta}_{2}$ can be written as

$$
V\left(\hat{\theta}_{1}, \hat{\theta}_{2}\right)=-\frac{1}{2}\left\{\int_{\underline{\theta}}^{\hat{\theta}_{1}}\left(\hat{\theta}_{1}-\theta-\delta\right)^{2} f(\theta) d \theta+\delta^{2} \int_{\hat{\theta}_{1}}^{\hat{\theta}_{2}} f(\theta) d \theta+\int_{\hat{\theta}_{2}}^{\bar{\theta}}\left(\hat{\theta}_{2}-\theta-\delta\right)^{2} f(\theta) d \theta\right\} .
$$

\footnotetext{
${ }^{9}$ Again $q(x, \varepsilon) \in\{x-\Delta, x+\Delta\}$.

${ }^{10}$ If $x+\Delta>\bar{\theta}$ or $x-\Delta<\underline{\theta}$ then in (5) $x+\Delta$ is replaced by $\bar{\theta}$ and $x-\Delta$ is replaced by $\underline{\theta}$ correspondingly.
} 
Optimizing with respect to $\hat{\theta}_{2}$ with the constraint $\hat{\theta}_{2} \geq \hat{\theta}_{1}$ yields

$$
\frac{\partial V}{\partial \hat{\theta}_{2}}\left(\hat{\theta}_{1}, \hat{\theta}_{2}\right)=-\int_{\hat{\theta}_{2}}^{\bar{\theta}}\left(\hat{\theta}_{2}-\theta-\delta\right) f(\theta) d \theta>0
$$

so that $\hat{\theta}_{2}=\bar{\theta}$ and we can rewrite $V(\cdot)$ as a function of $\hat{\theta}_{1}$ only. Abusing slightly notations, we have:

$$
V\left(\hat{\theta}_{1}\right)=-\frac{1}{2}\left\{\int_{\underline{\theta}}^{\hat{\theta}_{1}}\left(\hat{\theta}_{1}-\theta-\delta\right)^{2} f(\theta) d \theta+\delta^{2}\left(1-F\left(\hat{\theta}_{1}\right)\right)\right\} .
$$

The first-order condition $\left.\frac{d}{d \hat{\theta}_{1}} V\left(\hat{\theta}_{1}\right)\right|_{\hat{\theta}_{1}=\hat{\theta}}=0$ yields the expression of $\hat{\theta}$ given by $(6)$.

Moreover, we have

$$
\frac{d^{2}}{d \hat{\theta}_{1}} V\left(\hat{\theta}_{1}\right)=\delta f\left(\hat{\theta}_{1}\right)-F\left(\hat{\theta}_{1}\right)
$$

and thus the second-order condition

$$
\left.\frac{d^{2}}{d \hat{\theta}_{1}} V\left(\hat{\theta}_{1}\right)\right|_{\hat{\theta}_{1}=\hat{\theta}}=\delta f(\hat{\theta})-F(\hat{\theta}) \leq 0
$$

is satisfied if and only if

$$
\frac{f(\hat{\theta})}{F^{2}(\hat{\theta})} \int_{\underline{\theta}}^{\hat{\theta}} F(\theta) d \theta \leq 1 .
$$

However, consider the function $\varphi(\theta)=\frac{1}{F(\theta)} \int_{\underline{\theta}}^{\theta} F(x) d x$. We have

$$
\dot{\varphi}(\theta)=1-\frac{f(\theta)}{F^{2}(\theta)} \int_{\underline{\theta}}^{\theta} F(x) d x=1-\frac{f(\theta)}{F(\theta)} \varphi(\theta) .
$$

But when $F(\cdot)$ is log-concave, $\frac{d}{d \theta}\left(\frac{F(\theta)}{f(\theta)}\right)>0$ and

$$
\varphi(\theta)=\frac{1}{F(\theta)} \int_{\underline{\theta}}^{\theta} \frac{F(x)}{f(x)} f(x) d x \leq \frac{F(\theta)}{f(\theta)},
$$

so that $\dot{\varphi}(\theta) \geq 0$. Finally, (A4) holds. 